\title{
Enhanced Surveillance of Nonfatal Emergency Department Opioid Overdoses in California
}

\author{
Natalie Demeter, Jaynia A. Anderson, Mar-y-sol Pasquires, Stephen Wirtz
}

California Department of Public Health, Sacramento, California, United States

Objective

To track and monitor nonfatal emergency department opioid overdoses in California for use in the statewide response in the opioid epidemic.

\section{Introduction}

The opioid epidemic is a multifaceted public health issue that requires a coordinated and dynamic response to address the ongoing changes in the trends of opioid overdoses. Access to timely and accurate data allows more targeted and effective programs and policies to prevent and reduce fatal and nonfatal drug overdoses in California. As a part of a Centers for Disease Control and Prevention Enhanced State Opioid Overdose Surveillance grant, the goals of this surveillance are to more rapidly identify changes in trends of nonfatal drug overdose, opioid overdose, and heroin overdose emergency department visits; identify demographic groups or areas within California that are experiencing these changes; and to provide these data and trends to state and local partners addressing the opioid crisis throughout California. Emergency department (ED) visit data are analyzed on an ongoing quarterly basis to monitor the proportion of all ED visits that are attributed to nonfatal drug, opioid, and heroin overdoses as a portion of the statewide opioid overdose surveillance.

\section{Methods}

California emergency department data were obtained from the California Office of Statewide Health Planning and Development. Data were (and continue to be) analyzed by quarter as the data become available, starting in quarter 1 (Q1) 2016 through Q1 2018. Quarters were defined as standard calendar quarters; January-March (Q1), April-June (Q2), July-September (Q3), and OctoberDecember (Q4). Counts of nonfatal ED visits for all drug overdoses, all opioid overdoses, and heroin overdoses were defined by the following ICD-10 codes in the principle diagnosis or external cause of injury fields respectively; T36X-T50X (all drug), T40.0X-T40.4X T40.6 and T40.69 (all opioid), and T40.1X (heroin). Eligible ED visits were limited to CA residents, patients greater than 10 years of age, initial encounters, and were classified as unintentional overdoses or overdoses of undetermined intent. Overdose ED visits are described by quarter, drug, sex, and age for Q1 2016 - Q1 2018.

\section{Results}

On average, 6,450 emergency department visits in California are attributed to drug overdose every quarter. Between Q1 2016 and Q1 2018, on average 1,785 (range: 1,559-2,011 ED visits) of those visits were due to opioid overdoses and a further 924 (52\%) of those ED visits were due to heroin overdoses. About 26-30\% of all drug overdose ED visits were for opioid overdoses in California during Q1 2016 - Q1 2018. Quarterly, that is around 6.00-7.64 opioid overdose ED visits for every 10,000 ED visits (Table 1), with about half those (3.09-4.30 ED visits) being heroin overdose ED visits. Males accounted for approximately $52 \%$ of all drug overdose ED visits, $65 \%$ of all opioid overdose ED visits, and $76 \%$ of all heroin overdose ED visits per quarter. Across all quarters, 25-34 year olds had the highest proportion of emergency department visits attributed to opioid and heroin overdose compared to all other age groups. However, 11-24 year olds had the highest proportion of emergency department visits attributed to all drug overdoses compared to all other age groups for all quarters except one. Between Q1 2016 and Q1 2018, the proportion of emergency department visits attributed to all drug overdoses increased by $1.8 \%$, all opioid overdoses increased $3.1 \%$, and heroin overdoses increased by $13.5 \%$.

\section{Conclusions}

Overall trends for the proportion of all emergency department visits for all drug overdoses and all opioid overdoses are relatively stable over this time period, however the proportion of heroin overdose ED visits shows a more substantial increase between Q1 2016 and Q1 2018. In addition, heroin overdose ED visits account for over half of all opioid overdose ED visits during this time in California. Ongoing surveillance of drug, opioid, and heroin overdose ED visits is a crucial component of assessing and responding to the opioid overdose crisis in California and helps to better understand the demographics of those who could be at risk of a future

SDS Annual Conference Proceedings 2019. This is an Open Access article distributed under the terms of the Creative Commons AttributionNoncommercial 4.0 Unported License (http://creativecommons.org/licenses/by-nc/3.0/), permitting all non-commercial use, distribution, and reproduction in any medium, provided the original work is properly cited. 


\section{OJPHI}

ISDS 2019 Conference Abstracts

fatal opioid overdose. Timely data such as these (in addition to prescribing, hospitalization, and death data) can inform local and statewide efforts to reduce opioid overdoses and deaths.

\section{Acknowledgement}

This project was supported by the U.S. Centers for Disease Control and Prevention, NU17CE924903-02-00.

Table 1. Proportion of all CA Emergency Department Visits attributed to Drug Overdose (per 10,000 visits)

\begin{tabular}{|c|c|c|c|}
\hline $\begin{array}{c}\text { Quarter } \\
\text { and Year }\end{array}$ & All Drug & $\begin{array}{c}\text { All } \\
\text { Opioid }\end{array}$ \\
\hline Q1 2016 & 24.05 & 6.57 & 3.05 \\
\hline Q2 2016 & 26.19 & 6.77 & 3.09 \\
\hline Q3 2016 & 24.69 & 6.82 & 3.49 \\
\hline Q4 2016 & 24.55 & 7.00 & 3.56 \\
\hline Q1 2017 & 23.45 & 7.64 & 4.11 \\
\hline Q2 2017 & 25.73 & 6.73 \\
\hline Q3 2017 & 25.81 & 24.38 & 6.77 \\
\hline
\end{tabular}

